— \& Joseph, P. (1993) Remands to hospital for psychiatric reports: a study of psychiatrists' attitudes to section 35 of the Mental Health Act 1983. Psychiatric Bulletin, 17, 197-199.

Institute of Psychiatry

Denmark Hill

London SE5 8AF

Springfield Hospital

Glenburnie Road

London $S W 17$

\section{Integrated psychological therapy for schizophrenia}

SIR: It is disappointing that Brenner et al (Journal, October 1992, 161 (suppl. 18), 154-163) could not report more encouraging results for their Integrated Psychological Therapy Programme for Schizophrenic Patients (IPT). This is especially disappointing given Brenner's pioneering work in attending to the cognitive deficits in schizophrenia in rehabilitation programmes.

While "favourable overall effects of IPT" are reported, the effects of Brenner's Cognitive Differentiation Module on both lower-order and higher-order functioning are questioned. Significantly, in our view, the Cognitive Module did not result in improvement in card sorting (a variant of the Wisconsin Card Sorting Test) or in attention (as measured by the D2-test).

It is possible that the IPT Cognitive Differentiation Module was not administered effectively. The training was brief, and given to groups of patients. Individual, one-to-one training may be necessary if cognitive strategies are to be imparted; strategies which could generalise to higher-order activities. Additionally, and as pointed out in the conclusion of the paper, programmes may need to be "more firmly based on comprehensive cognitive theories".

A variant of the IPT was introduced to Australia in 1988. Gains were achieved in measures of cognitive functions (such as certain subtests of the WAIS-R), and there were improvements in symptoms (as measured by the Expanded Brief Psychiatric Rating Scale) and in the skills of daily living (as measured by the Life Skills Profile). However, there were no gains in cognitive flexibility (as measured by the Wisconsin Card Sorting Test), nor in forward planning (as measured by the Tower of London).

This led us to abandon the IPT, and to concentrate on neurocognitive rehabilitation development as a 'front-end' to higher-order training, such as Communication Skills, Psycho-education, and Living Skills Modules. A computerised package, developed for patients with brain damage following head injury or stroke (Bracy, 1987), was administered to seven schizophrenic patients. Gains were recorded similar to those for the modified IPT, but again, there were no improvements in cognitive flexibility or forward planning.

A neurocognitive rehabilitation programme based on putative prefrontal/frontal executive functions, as subserved by distributed neural networks, was then developed, and called the Frontal/Executive Program. Improvements were recorded as for the ITP and for the Bracy computerised programs but, in addition, significant improvements in cognitive flexibility and forward planning were achieved (Morice \& Delahunty, 1993).

Some 20 schizophrenic patients have now completed the Frontal/Executive Program, which comprises a two-week Cognitive Fexibility Module, and a six-week Planning Module, administered on a one-to-one basis. Results are encouraging, and suggest a generalisation to higher-order activities. Anecdotally, patients report clearer thinking and improved functioning, and their families quite often report marked changes in a range of behaviours.

We regard neurocognitive rehabilitation in schizophrenia as an important new adjunct to management, an innovation for which Brenner and colleagues are largely responsible. But possibly they have not been critical enough of their Cognitive Differentiation Module, have not developed it further on the basis of an adequate cognitive theory, and have not made sufficient modifications to it in the light of earlier results.

We encourage readers to remain more optimistic than the Bern originators of this approach to rehabilitation. While much more development and evaluation remains to be done, early results would seem to justify continuing optimism.

BrACY, O. L. (1987) Cognitive Rehabilitation Programs. Indianapolis: Psychological Software Services.

MORICE, R. D. \& DELAHUNTY, A. (1993) Treatment strategies for the remediation of neurocognitive dysfunction in schizophrenia. In The Neuropsychology of Schizophrenia (eds C. Pantelis, H. E. Nelson \& T. Barnes). Sussex: Wiley (in press)

RODNEY MORICE

Mental Health Unit

ANN DelahuntY

South West Region NSW

481 George Street

Albury NSW 2640

Australia

AUTHORS' REPLY: Morice \& Delahunty refer to the effects of IPT. IPT is a comprehensive group therapy consisting of five subprogrammes which proceed from functional cognitive skills to general behavioural 